\title{
Reacción al Fuego de Tres Maderas Angiospermas Impregnadas con Sales de Boro
}

\section{Reaction to fire of three angiosperm woods impregnated with boron salts}

\author{
Javier Ramón Sotomayor Castellanos 1 https://orcid.org/0000-0002-1527-8801 \\ Luz Elena Alfonsina Ávila Calderón ${ }^{1}$ https:///orcid.org/0000-0003-2646-2142 \\ ${ }^{1}$ Universidad Michoacana de San Nicolás de Hidalgo, Morelia, México \\ madera999@yahoo.com \\ lea.avilacalderonegmail.com
}

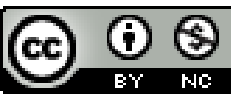

Esta obra está bajo una licencia internacional Creative Commons Atribución-NoComercial 4.0
Enviado:

Aceptado:

Publicado:
$2021 / 04 / 27$

$2021 / 05 / 20$

$2021 / 06 / 30$

\section{Resumen}

La madera es un material combustible, motivo por el cual es importante investigar técnicas para aumentar su resistencia al fuego. La retención de sales de boro en la madera mide la idoneidad del tratamiento de impregnación y la aptitud que tiene una especie para su preservación y reacción al fuego. Los objetivos de la investigación fueron, por una parte, determinar su densidad aparente y su capacidad de retención de sales de boro y, por otra, medir el tiempo de ignición y la pérdida de masa en pruebas de reacción al fuego. La investigación comprobó la capacidad de retención de sales de boro de Spathodea campanulata (Tulipán africano), Fraxinus americana (Fresno blanco) y Albizia plurijuga (Tepehuaje). Para cada especie, se impregnaron cuarenta probetas de pequeñas dimensiones con el método de baño caliente-frío con una solución de ácido bórico y borato de sodio con concentraciones de uno, dos y tres por ciento. Se determinaron la densidad y el contenido de humedad de la madera. Se realizaron pruebas de reacción al fuego con duración de dos minutos y se midieron el tiempo de ignición y la pérdida de masa. El impregnado de la madera con sales de boro aumenta la resistencia aparente de la madera a la exposición al fuego. A medida que la densidad de la madera aumenta, los tiempos de ignición se incrementan y, a la vez, se disminuye la pérdida de masa.

Palabras clave: impregnación de la madera, retención, densidad aparente, tiempo de ignición, pérdida de masa.

\footnotetext{
Abstract

Wood is a combustible material; therefore, it is important to investigate techniques to increase its resistance to fire. The retention of boron salts in wood measures the suitability of the impregnation treatment and the aptitude of one species for its preservation and reaction to fire. The objectives of this

Sumario: 1 Introducción, 2 Materiales y Métodos, 3 Resultados y Discusión, 4 Conclusiones.

Como citar: Sotomayor Castellanos, J. R., \& Ávila Calderón, L. E. A. (2021). Reacción al Fuego de Tres Maderas Angiospermas Impregnadas con Sales de Boro. Revista Tecnológica - Espol, 33(1), 35-48.

http://www.rte.espol.edu.ec/index.php/tecnologica/article/view/818
} 
research are, on one hand, to determine the apparent density of this kind of wood and its capacity to retain boron salts and on the other hand, to measure ignition time and mass loss in reaction to fire tests. The investigation verified the capacity of retention of boron salts of Spathodea campanulata (Tulipán africano), Fraxinus americana (Fresno blanco) and Albizia plurijuga (Tepehuaje). For each species, forty small specimens were impregnated with the hot-cold bath method with a solution of boric acid and sodium borate with concentrations of one, two, and three percent. The density and moisture content of the wood were determined. Fire reaction tests were conducted lasting two minutes and the ignition time and mass loss were measured. Impregnated wood with boron salts increases the apparent resistance of wood to fire exposure. The density of the wood increases with the increase of ignition time whereas mass loss decreases.

Keywords: Wood impregnation, retention, apparent density, ignition time, mass loss.

\section{Introducción}

La madera es un material combustible, motivo por el cual arquitectos, diseñadores de elementos de construcción y fabricantes de muebles se han preocupado por indagar sobre las técnicas que permiten aumentar la resistencia de esta al fuego (Atar et al., 2011; Bartlett et al., 2019). Para tal propósito, se han desarrollado diferentes enfoques de investigación. Por ejemplo, aquel que efectúa pruebas con configuraciones que simulan condiciones reales de incendio para así poder recomendar criterios de diseño y cálculo estructural (Östman, 2017; Ali et al., 2019). Otro enfoque, en cambio, prefiere realizar pruebas de laboratorio con pequeñas probetas, con el objetivo de estudiar las variables que intervienen en la fenomenología de la reacción de la madera al fuego (Harada, 2001; Osvaldová, 2018). Otra perspectiva alude a las sustancias preservantes y los métodos de impregnación con el fin de, a partir de la caracterización del fenómeno de ignición de la madera, disminuir su deterioro cuando se expone al fuego (Tondi et al., 2012; Wang et al., 2017 Gaff et al., 2019).

La medición de los niveles de retención de sales de boro en la madera facilita establecer la idoneidad de un determinado tratamiento de impregnación y la aptitud que tiene una especie para preservarse del fuego (Koumbi-Mounanga et al., 2015, Pereira et al., 2017). Existe evidencia empírica de que, si una probeta de madera de pequeñas dimensiones es impregnada con sales de boro y expuesta al fuego, el tiempo de ignición y la pérdida de masa disminuyen (Sogutlu et al., 2011; Uner et al., 2016; Jin \& Chung, 2018). En este proceso intervienen, entre otros factores, la especie, su densidad aparente, la retención de la sustancia preservante, así como las condiciones de laboratorio en las cuales se realizan las pruebas de reacción al fuego (Babrauskas, 2002; Osvaldová et al., 2016). La información revisada sobre el tema en la literatura es amplia y los resultados hallados en esta son diferentes en cada caso.

Como ya se mencionó, uno de los factores que influye en el momento de evaluar el comportamiento de la madera expuesta al fuego es la densidad aparente, porque cuando esta aumenta el tiempo de ignición también se incrementa (Osvaldová, 2018). Por su parte, el tiempo de ignición, que es un factor importante para evaluar el comportamiento de combustión de los materiales, se define como el lapso requerido para percibir la flama sostenida en la superficie de una muestra expuesta a un flujo de calor incidente, y, cuanto más corto sea el tiempo de ignición, más inflamable será el material (Boonmee \& Quintiere, 2002; Xu et al., 2015).

Entre tanto, la pérdida de masa, como resultado de la exposición de la madera al fuego, puede servir como indicador de la vocación de una especie para su empleo en estructuras y productos. La literatura sobre el comportamiento de la madera expuesta al fuego establece que la pérdida de masa es proporcional a la densidad de la madera (Friquin, 2011; Rocha \& Landesmann, 2016) y que la pérdida de masa depende, principalmente, del tipo de especie maderable, de la temperatura y el tiempo de exposición al calor ígneo (Ragan et al., 2016).

P. Beauv ha estudiado las características tecnológicas de la especie Spathodea campanulata. Britton y Rose, además de otros autores, como Silva et al. (2010), Sotomayor (2015) y Tamarit y López (2017), han estudiado las especies Fraxinus americana y Albizia plurijuga (Standl.). Sin embargo, no se detectó información sobre su reacción al fuego en un contexto en el que estas especies hayan sido 
impregnadas previamente con sales de boro. Los objetivos de la investigación fueron, por una parte, determinar su densidad aparente y su capacidad de retención de sales de boro y, por otra, medir el tiempo de ignición y la pérdida de masa en pruebas de reacción al fuego.

\section{Materiales y Métodos}

Las piezas de madera de S. campanulata, F. americana y A. plurijuga se recolectaron en aserraderos del Estado de Michoacán, México. Para cada especie se prepararon 40 probetas, con dimensiones de $0,02 \mathrm{~m}$ x 0,02 m de sección y 0,4 m de largo, orientadas, respectivamente, en las direcciones radial, tangencial y longitudinal de la madera, tal como lo estipula la norma ISO 3129:2012 (International Organization for Standardization, 2012). Las probetas contenían solo madera de duramen, libre de defectos de crecimiento. Se almacenaron en una cámara de acondicionamiento durante 12 meses, con una temperatura de $20{ }^{\circ} \mathrm{C}\left( \pm 1^{\circ} \mathrm{C}\right)$ y una humedad relativa del aire de $65 \%( \pm 2$ $\%)$ hasta que su peso alcanzó un valor constante. El material experimental de esta investigación corresponde al mismo tipo que Sotomayor y Ávila utilizaron y reportaron (2019) en experimentos que se realizaron de manera paralela a la presente investigación.

El contenido de humedad se calculó en un grupo adicional de 20 muestras, obtenidas del recorte que se efectuó, después de las pruebas, en un extremo de las probetas, con dimensiones de $0,02 \mathrm{~m} \times$ $0,02 \mathrm{~m} \times 0,06$. El contenido de humedad se determinó por el método de diferencia de pesos, que realiza su cálculo de acuerdo con la norma ISO 13061-1:2014 (International Organization for Standardization, 2014a). La densidad aparente de la madera se determinó de acuerdo con la norma ISO 13061-2:2014 (International Organization for Standardization, 2014b). Cabe señalar que, para aligerar el texto cuando conviene, se ha utilizado el término "densidad" en lugar del concepto "densidad aparente".

\section{Proceso de impregnación}

Las probetas se impregnaron con el método baño caliente-frío, siguiendo el protocolo propuesto por Ávila et al. (2012). Se prepararon 30 litros de solución de trihidróxido de boro (ácido bórico, 39,4 $\%$ ) y tetraborato de sodio (borato de sodio, 60,6 \%) con tres concentraciones: $1 \%, 2 \%$ y $3 \%$, tal como lo establece la norma NMX-C-178-ONNCCE-2014 (Organismo Nacional de Normalización y Certificación de la Construcción y Edificación, 2014). La madera se sumergió durante 8 h en un baño de agua con temperatura de $60{ }^{\circ} \mathrm{C}$ y presión atmosférica. A continuación, las probetas se sumergieron durante $16 \mathrm{~h}$ en un baño frío, con la solución de sales de boro a temperatura de $23{ }^{\circ} \mathrm{C}$ y presión atmosférica.

La retención se determinó con la fórmula (1) (Simsek et al., 2013):

$$
\mathrm{R}=\frac{\left(\mathrm{P}_{\mathrm{DT}}-\mathrm{P}_{\mathrm{AT}}\right) \times \mathrm{C}}{\mathrm{V}_{\mathrm{DT}}}
$$

Donde:

$\mathrm{R}=$ Retención $\left(\mathrm{kg} \mathrm{m}^{-3}\right)$

$\mathrm{P}_{\mathrm{DT}}=$ Peso después del tratamiento de impregnación $(\mathrm{kg})$

$\mathrm{P}_{\mathrm{AT}}=$ Peso antes del tratamiento de impregnación $(\mathrm{kg})$

$\mathrm{C}=$ Concentración de sales de boro (\%)

$\mathrm{V}_{\mathrm{DT}}=$ Volumen después del tratamiento de impregnación $\left(\mathrm{m}^{3}\right)$

\section{Pruebas de reacción al fuego}

Las pruebas de reacción al fuego se llevaron a cabo según el protocolo reportado por Sotomayor y Carrillo (2017). La metodología implementada para dichas pruebas se dividió en las siguientes etapas:

1. Se midieron el peso y las dimensiones de la probeta antes del tratamiento.

2. La probeta se posicionó en el dispositivo para las pruebas de comportamiento al fuego (Figura 1). Su orientación siguió la dirección radial, lo que coincidió con el flujo vertical de la flama. 
3. La probeta se expuso durante 2 minutos al fuego directo de la flama de un mechero de laboratorio tipo Meker-Fisher, con regulación de aire y de gas, quemador de 0,03 m de diámetro y temperatura máxima de $1300{ }^{\circ} \mathrm{C}$.

4. Con un cronómetro se midió el tiempo de ignición (tign) definido de acuerdo con el tiempo que transcurrió hasta que la ignición apareció en al menos tres de las caras de la probeta expuestas a la flama.

5. La probeta se retiró de la flama y se interrumpió su combustión en una cama de arena.

6. La masa de la zona carbonizada se retiró y, luego, se procedió a medir el peso de la probeta después del tratamiento.

\section{Figura 1}

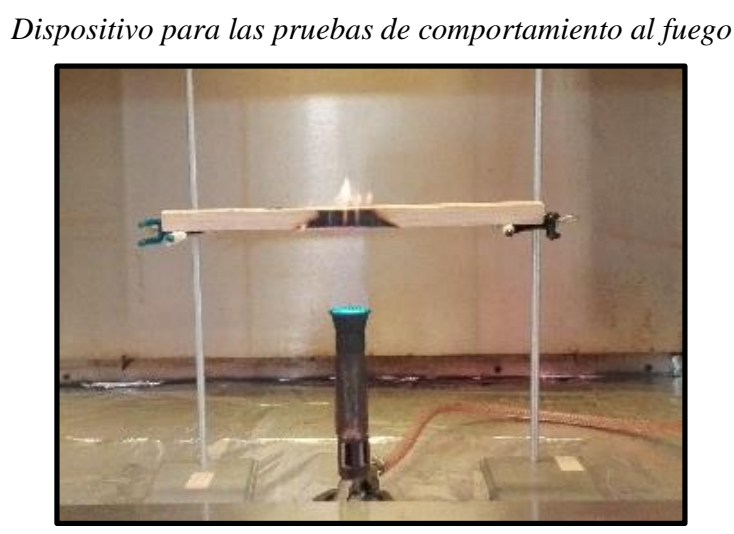

La pérdida de masa se determinó con la fórmula (2) (Gaff et al., 2019):

$$
\Delta \mathrm{m}=\frac{\mathrm{P}_{\mathrm{AT}}-\mathrm{P}_{\mathrm{DT}}}{\mathrm{P}_{\mathrm{AT}}} \times 100
$$

Donde:

$\Delta \mathrm{m}=$ Pérdida de masa $(\%)$

$\mathrm{P}_{\mathrm{AT}}=$ Peso antes de la exposición al fuego $(\mathrm{kg})$

$\mathrm{P}_{\mathrm{DT}}=$ Peso después de la exposición al fuego $(\mathrm{kg})$

\section{Diseño experimental}

Se examinaron tres unidades experimentales, que se organizaron en torno a cada una de las siguientes especies: S. campanulata, $F$. americana y A. plurijuga. El factor de variación fue el tratamiento de impregnación con cuatro niveles de concentración de sales de boro: $1 \%, 2 \%$, y $3 \%$. El grupo de control fue el del nivel cero, y no se le dio tratamiento. Para cada especie y cada concentración, se efectuaron muestras independientes de diez probetas cada una, por lo que en total se analizaron 120 probetas $(3 \times 4 \times 10)$. Las variables de respuesta fueron la densidad aparente $\left(\rho_{\mathrm{CH}}\right)$, la retención de sales de boro $(\mathrm{R})$, el tiempo de ignición $\left(\mathrm{t}_{\mathrm{ign}}\right)$ y la pérdida de masa $(\Delta \mathrm{m})$. El contenido de humedad $(\mathrm{CH})$ se consideró variable de referencia y no intervino en los resultados.

Para cada variable se calculó su media $(\overline{\mathrm{x}})$, su desviación estándar $(\sigma)$ y su coeficiente de variación porcentual $(\mathrm{CV}=\sigma / \overline{\mathrm{x}})$. Se determinó su normalidad en la distribución con el criterio de demarcación para el sesgo estandarizado (SE) y para el apuntamiento estandarizado (AE): [-2 < SE y/o $\mathrm{AE}<+2$ ]; se verificaron la igualdad de varianzas (Ver-var) con la hipótesis nula: $\mathrm{H}_{0}: \sigma_{1}=\sigma_{2}$, con un nivel de significancia de $5 \%(\alpha=0,05)$. Luego, se practicaron análisis de varianza (Anova) con la hipótesis nula: $\mathrm{H}_{0}: \overline{\mathrm{x}}_{1}=\overline{\mathrm{x}}_{2}$, con un nivel de significancia de $5 \%(\alpha=0,05)$. El criterio de demarcación fue aceptar una diferencia estadísticamente significativa para valores $\mathrm{P}_{(\alpha=0,05)}<0,05$. Cuando no se confirmó la normalidad en la distribución de las muestras, se realizaron pruebas no paramétricas de 
Kruskal-Wallis de diferencia de medianas $(\overline{\mathrm{X}})$ para un nivel de confiabilidad del $95 \%$. Para las pruebas no paramétricas, las hipótesis resultaron similares, pero se emplearon las medianas.

\section{Resultados y Discusión}

Las maderas de $S$. campanulata, $F$. americana y A. plurijuga alcanzaron un contenido de humedad equilibrado, con un promedio de $10 \%$, un coeficiente de variación de $8 \%$. Estos parámetros indican que la madera se acondicionó a un estado de humedad seco y homogéneo, de tal forma que la variación en el contenido de humedad de la madera no intervino de modo significativo en los resultados.

El impregnado de la madera con sales de boro sí aumentó la resistencia aparente de la madera a la exposición del fuego. A medida que la concentración de sales de boro se incrementó, la pérdida de masa en la zona de combustión disminuyó (Figura 2).

Figura 2

Disminución de la sección transversal en función de la concentración $(C)$ en probetas de A. plurijuga

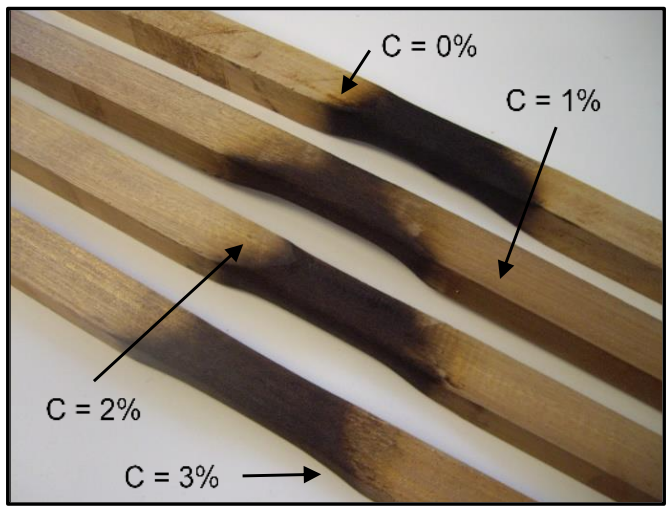

\section{Densidad}

Las magnitudes de las densidades de la madera sin tratamiento $(\mathrm{C}=0 \%)$ de $S$. campanulata, F. americana y A. plurijuga (Tabla 1) son similares a las reportadas en la bibliografía (Silva et al., 2010; Sotomayor, 2015; Tamarit \& López, 2017). En el siguiente cuadro (Tabla 1), las densidades correspondientes a cada especie siguen el ordenamiento: $S$. campanulata $>$ F. americana $>$ A. plurijuga. Además, evidencia que los coeficientes de variación de la densidad de la madera sin tratamiento aumentan o disminuyen dependiendo de la especie o de la concentración. No se observa una tendencia definida hacia la disminución, pero tampoco se advierte un aumento desproporcional ocasionado por el tratamiento.

Tabla 1

Resultados de las pruebas de exposición al fuego

\begin{tabular}{|c|c|c|c|c|c|}
\hline $\mathbf{C}$ & & $\rho_{\mathrm{CH}}$ & $\mathbf{R}$ & $t_{\text {ign }}$ & $\Delta \mathbf{m}$ \\
\hline \multirow[t]{2}{*}{$(\%)$} & & $\left(\mathrm{kg} \mathrm{m}^{-3}\right)$ & $\left(\mathrm{kg} \mathrm{m}^{-3}\right)$ & (s) & $(\%)$ \\
\hline & \multicolumn{5}{|c|}{ S. campanulata } \\
\hline \multirow[t]{2}{*}{0} & $\overline{\mathrm{x}}$ & 351 & 0 & 12,7 & 6,0 \\
\hline & $\mathrm{CV}$ & $(6,3)$ & - & $(36,2)$ & $(30,2)$ \\
\hline \multirow[t]{2}{*}{1} & $\overline{\mathrm{x}}$ & 351 & 2,3 & 11,3 & 6,4 \\
\hline & $\mathrm{CV}$ & $(6,4)$ & $(4,6)$ & $(30,1)$ & $(26,5)$ \\
\hline \multirow[t]{2}{*}{2} & $\overline{\mathrm{x}}$ & 336 & 4,2 & 12,6 & 6,4 \\
\hline & $\mathrm{CV}$ & $(3,9)$ & $(4,8)$ & $(21,6)$ & $(9,3)$ \\
\hline \multirow[t]{3}{*}{3} & $\overline{\mathrm{x}}$ & 340 & 6,4 & 11,1 & 6,4 \\
\hline & $\mathrm{CV}$ & $(8,5)$ & $(6,4)$ & $(10,8)$ & $(4,6)$ \\
\hline & \multicolumn{5}{|c|}{ F. americana } \\
\hline \multirow[t]{2}{*}{0} & $\overline{\mathrm{x}}$ & 654 & 0 & 11,6 & 6,2 \\
\hline & $\mathrm{CV}$ & $(5,6)$ & - & $(13,6)$ & $(6,7)$ \\
\hline 1 & $\overline{\mathrm{x}}$ & 616 & 1,6 & 13,7 & 5,8 \\
\hline
\end{tabular}




\begin{tabular}{|c|c|c|c|c|c|}
\hline & $(\mathrm{CV}$ & $(3,6)$ & $(9,5)$ & $(1,6)$ & $(9,1)$ \\
\hline \multirow[t]{2}{*}{2} & $\overline{\mathrm{x}}$ & 619 & 3,3 & 17,0 & 5,7 \\
\hline & $\mathrm{CV}$ & $(7,5)$ & $(6,3)$ & $(11,1)$ & $(13,7)$ \\
\hline \multirow[t]{3}{*}{3} & $\overline{\mathrm{x}}$ & 610 & 5,3 & 21,5 & 5,5 \\
\hline & $\mathrm{CV}$ & $(4,8)$ & $(10,2)$ & $(7,7)$ & $(10,0)$ \\
\hline & \multicolumn{5}{|c|}{ A. plurijuga } \\
\hline \multirow[t]{2}{*}{0} & $\overline{\mathrm{x}}$ & 830 & 0 & 15,0 & 4,4 \\
\hline & $\mathrm{CV}$ & $(2,7)$ & - & $(15,7)$ & $(8,8)$ \\
\hline \multirow[t]{2}{*}{1} & $\overline{\mathrm{x}}$ & 831 & 1,3 & 19,3 & 3,9 \\
\hline & $\mathrm{CV}$ & $(2,2)$ & $(9,8)$ & $(14,7)$ & $(6,7)$ \\
\hline \multirow[t]{2}{*}{2} & $\overline{\mathrm{x}}$ & 846 & 2,7 & 20,6 & 4,1 \\
\hline & $\mathrm{CV}$ & $(5,0)$ & $(5,9)$ & $(12,8)$ & $(7,8)$ \\
\hline \multirow[t]{2}{*}{3} & $\overline{\mathrm{x}}$ & 816 & 3,7 & 25,3 & 3,9 \\
\hline & $\mathrm{CV}$ & $(2,8)$ & $(7,3)$ & $(5,9)$ & $(2,5)$ \\
\hline
\end{tabular}

$\rho_{\mathrm{CH}}=$ Densidad; $\mathrm{R}=$ Retención; $\mathrm{t}_{\mathrm{ign}}=$ Tiempo de ignición; $\Delta \mathrm{m}=$ Pérdida de masa; $\mathrm{C}=$ Concentración; $\overline{\mathrm{x}}=\mathrm{Media} ; \mathrm{CV}=\mathrm{Coeficiente}$ de variación en porciento y entre paréntesis.

Fuente: Elaboración propia

Las distribuciones de todas las muestras resultaron normales (Tabla 2), con excepción de las de S. campanulata sin tratamiento $(\mathrm{C}=0 \%$ : $\mathrm{SE}$ y $\mathrm{AE}>2)$. Las pruebas de verificación y análisis de varianza no muestran diferencias estadísticamente significativas $\left(\mathrm{P}_{(\alpha=0,05)}>0,05\right)$ para $S$. campanulata y A. plurijuga (Tabla 3). Un caso diferente corresponde a las densidades de $F$. americana, las cuales muestran una diferencia significativa $\left(\mathrm{P}_{(\alpha=0,05)}<0,05\right)$. Las pruebas de grupos homogéneos indican que, para $S$. campanulata, las densidades, según las concentraciones (grupos de muestras en la Tabla 3), son homogéneas entre ellas. En cambio, para $F$. americana y A. plurijuga se agrupan y combinan en diferentes concentraciones.

\section{Tabla 2}

Resultados de las pruebas de normalidad

\begin{tabular}{|c|c|c|c|c|c|c|c|c|}
\hline & \multicolumn{2}{|c|}{$\mathrm{C}=0 \%$} & \multicolumn{2}{|c|}{$\mathrm{C}=1 \%$} & \multicolumn{2}{|c|}{$\mathrm{C}=2 \%$} & \multicolumn{2}{|c|}{$\mathrm{C}=3 \%$} \\
\hline & SE & $\mathbf{A E}$ & SE & $\mathbf{A E}$ & SE & $\mathbf{A E}$ & SE & $\mathbf{A E}$ \\
\hline & \multicolumn{8}{|c|}{ S. campanulata } \\
\hline$\rho_{\mathrm{CH}}$ & 2,6840 & 2,8500 & $-0,5667$ & $-0,5415$ & $-0,0072$ & 0,3682 & 0,5001 & 0,0054 \\
\hline $\mathrm{R}$ & - & - & $-0,1245$ & 0,4658 & $-0,6149$ & $-0,4818$ & 0,0429 & 1,3880 \\
\hline$t_{\text {ign }}$ & 1,6960 & 1,2850 & $-0,0591$ & $-1,1030$ & 0,5755 & $-0,7850$ & $-0,3009$ & $-0,2379$ \\
\hline \multirow[t]{2}{*}{$\Delta \mathrm{m}$} & 1,7510 & 0,5705 & $-0,8481$ & 0,3336 & 1,3910 & 1,4280 & 0,7590 & 0,4514 \\
\hline & \multicolumn{8}{|c|}{ F. americana } \\
\hline$\rho_{\mathrm{CH}}$ & 0,2382 & $-1,2460$ & 0,4932 & $-0,2227$ & 0,2952 & $-0,6957$ & $-0,8263$ & $-0,9480$ \\
\hline $\mathrm{R}$ & - & - & $-0,2723$ & 0,1108 & $-0,2013$ & $-0,6870$ & 0,7573 & $-0,6038$ \\
\hline $\mathrm{t}_{\mathrm{ign}}$ & $-0,2959$ & $-0,5296$ & 0,0393 & $-0,4225$ & $-0,8023$ & 1,1660 & 1,0780 & 0,9941 \\
\hline \multirow[t]{2}{*}{$\Delta \mathrm{m}$} & 1,8630 & 0,3033 & $-0,0572$ & $-0,5249$ & 0,9110 & $-0,1152$ & 0,2073 & $-0,7578$ \\
\hline & \multicolumn{8}{|c|}{ A. plurijuga } \\
\hline$\rho_{\mathrm{CH}}$ & $-0,0899$ & $-0,8442$ & $-0,5070$ & $-0,5870$ & 0,0227 & $-0,1543$ & $-0,8722$ & 0,5468 \\
\hline $\mathrm{R}$ & - & - & $-0,1431$ & $-0,7043$ & 0,5075 & $-0,6602$ & $-1,6640$ & 1,1280 \\
\hline$t_{\text {ign }}$ & 1,725 & 0,6590 & 1,0740 & 0,1032 & 1,3510 & 0,6334 & $-0,8252$ & $-0,6403$ \\
\hline$\Delta \mathrm{m}$ & $-0,3254$ & $-0,1120$ & $-0,6241$ & $-0,7839$ & $-0,7292$ & 1,2100 & $-0,7764$ & 0,2558 \\
\hline
\end{tabular}

$\mathrm{C}=$ Concentración; $\mathrm{SE}=$ Sesgo estandarizado; $\mathrm{AE}=$ Apuntalamiento estandarizado; $\rho_{\mathrm{CH}}=$ Densidad; $\mathrm{R}=\mathrm{Retención} ; \mathrm{t}_{\mathrm{ign}}=\mathrm{Tiempo}$ de ignición; $\Delta \mathrm{m}=$ Pérdida de masa.

Fuente: Elaboración propia

\section{Retención}

Los resultados de las retenciones de sales de boro obtenidos en esta investigación (Tabla 1) son similares a los reportados en la bibliografía sobre especies mexicanas en las que se han aplicado protocolos experimentales parecidos a los de esta investigación. Sotomayor y Correa (2016) reportan para $S$. campanulata $\left(\rho_{\mathrm{CH}}=357 \mathrm{~kg} \mathrm{~m}^{-3}\right)$ una retención de $14,31 \mathrm{~kg} \mathrm{~m}^{-3}$; para A. religiosa $\left(\rho_{\mathrm{CH}}=419 \mathrm{~kg}\right.$ $\left.\mathrm{m}^{-3}\right)$, una retención de 7,23\%; y para G. ulmifolia $\left(\rho_{\mathrm{CH}}=739 \mathrm{~kg} \mathrm{~m}^{-3}\right)$, una retención de $9,7 \%$. Por su parte, Sotomayor y Villaseñor (2016) reportan valores de retención que varían en el intervalo de 4,07 $\%$ hasta 14,18 \% para diez especies mexicanas: Tilia mexicana, Cupressus lindley, Alnus acuminata, Cedrela odorata, Fraxinus uhdei, Lysiloma bahamensis, Fagus mexicana, Caesalpinia granadillo, 
Platymiscium dimorphandrum y Quercus spp. Los coeficientes de variación de las retenciones aumentan a medida que las concentraciones se incrementan para $S$. campanulata, $F$. americana y $A$. plurijuga, (Tabla 1). No se observa en estos una disposición relacionada con la especie o con la densidad.

Tabla 3

Resultados de las pruebas estadísticas

\begin{tabular}{|c|c|c|c|c|c|c|c|c|}
\hline & Ver-var & Anova & $\mathbf{K}-\mathbf{W}$ & $\mathbf{C}$ & \multicolumn{4}{|c|}{ Grupos homogéneos } \\
\hline & $P(\alpha=0,05)$ & $P(\alpha=0.05)$ & $\mathrm{P}(\alpha=0.05)$ & $(\%)$ & & & & \\
\hline & \multicolumn{8}{|c|}{ S. campanulata } \\
\hline$\rho_{\mathrm{CH}}$ & 0,4344 & - & 0.2996 & 0 & $\mathrm{X}$ & & & \\
\hline & - & - & - & 1 & $\mathrm{X}$ & & & \\
\hline & - & - & - & 2 & $X$ & & & \\
\hline & - & - & - & 3 & $\mathrm{X}$ & & & \\
\hline \multirow[t]{4}{*}{$\mathrm{R}$} & 0,1111 & $<0.0001$ & - & 0 & $\mathrm{X}$ & & & \\
\hline & - & - & - & 1 & & $\mathrm{X}$ & & \\
\hline & - & - & - & 2 & & & $\mathrm{X}$ & \\
\hline & - & - & - & 3 & & & & $\mathrm{X}$ \\
\hline \multirow[t]{4}{*}{$\mathrm{t}_{\mathrm{ign}}$} & 0,1080 & 0.5679 & - & 0 & $\mathrm{X}$ & & & \\
\hline & - & - & - & 1 & $\mathrm{X}$ & & & \\
\hline & - & - & - & 2 & $\mathrm{X}$ & & & \\
\hline & - & - & - & 3 & $\mathrm{X}$ & & & \\
\hline \multirow[t]{5}{*}{$\Delta \mathrm{m}$} & 0,0703 & 0.8787 & - & 0 & $X$ & & & \\
\hline & - & - & - & 1 & $X$ & & & \\
\hline & - & - & - & 2 & $\mathrm{X}$ & & & \\
\hline & - & - & - & 3 & $\mathrm{X}$ & & & \\
\hline & \multicolumn{8}{|c|}{ F. americana } \\
\hline \multirow[t]{4}{*}{$\rho_{\mathrm{CH}}$} & 0,1451 & 0.0312 & - & 0 & $\mathrm{X}$ & & & \\
\hline & - & - & - & 1 & $\mathrm{X}$ & & & \\
\hline & - & - & - & 2 & $X$ & & & \\
\hline & - & - & - & 3 & & $X$ & & \\
\hline \multirow[t]{4}{*}{$\mathrm{R}$} & 0,0003 & - & $<0.0001$ & 0 & $\mathrm{X}$ & & & \\
\hline & - & - & - & 1 & & $X$ & & \\
\hline & - & - & - & 2 & & & $\mathrm{X}$ & \\
\hline & - & - & - & 3 & & & & $\mathrm{X}$ \\
\hline \multirow[t]{4}{*}{$t_{\mathrm{ign}}$} & 0,9857 & $<0.0001$ & - & 0 & $\mathrm{X}$ & & & \\
\hline & - & - & - & 1 & & $\mathrm{X}$ & & \\
\hline & - & - & - & 2 & & & $\mathrm{X}$ & \\
\hline & - & - & - & 3 & & & & $\mathrm{X}$ \\
\hline \multirow[t]{5}{*}{$\Delta \mathrm{m}$} & 0,2446 & 0.0718 & - & 0 & $\mathrm{X}$ & & & \\
\hline & - & - & - & 1 & $\mathrm{X}$ & $X$ & & \\
\hline & - & - & - & 2 & $\mathrm{X}$ & $\mathrm{X}$ & & \\
\hline & - & - & - & 3 & & $X$ & & \\
\hline & \multicolumn{8}{|c|}{ A. plurijuga } \\
\hline \multirow[t]{4}{*}{$\rho_{\mathrm{CH}}$} & 0,1162 & 0.1403 & - & 0 & $\mathrm{X}$ & & & \\
\hline & - & - & - & 1 & $X$ & $X$ & & \\
\hline & - & - & - & 2 & $X$ & $\mathrm{X}$ & & \\
\hline & - & - & - & 3 & & $\mathrm{X}$ & & \\
\hline \multirow[t]{4}{*}{$\mathrm{R}$} & 0,0244 & - & $<0.0001$ & 0 & $X$ & & & \\
\hline & - & - & - & 1 & & $\mathrm{X}$ & & \\
\hline & - & - & - & 2 & & & $\mathrm{X}$ & \\
\hline & - & - & - & 3 & & & & $\mathrm{X}$ \\
\hline \multirow[t]{4}{*}{$t_{\text {ign }}$} & 0,4610 & $<0.0001$ & - & 0 & $X$ & & & \\
\hline & - & - & - & 1 & & $X$ & & \\
\hline & - & - & - & 2 & & $X$ & & \\
\hline & - & - & - & 3 & & & $\mathrm{X}$ & \\
\hline \multirow[t]{4}{*}{$\Delta \mathrm{m}$} & 0,0849 & 0.0017 & - & 0 & $\mathrm{X}$ & & & \\
\hline & - & - & - & 1 & $X$ & & & \\
\hline & - & - & - & 2 & $X$ & $X$ & & \\
\hline & - & - & - & 3 & & $X$ & & \\
\hline
\end{tabular}

Las distribuciones de los resultados de las retenciones del grupo de control y de las muestras de las tres concentraciones resultaron normales, con excepción de la retención alcanzada con la concentración de $2 \%$ para A. plurijuga (Tabla 2). Sin embargo, los valores medios de la retención presentan diferencias estadísticamente significativas de acuerdo con el nivel de concentración de sales 
de boro y entre especies (Tabla 3). En el mismo contexto, las pruebas de rangos múltiples indican que, para las tres especies en estudio, existen diferencias entre los cuatro grupos analizados.

Las retenciones de sales de boro en $S$. campanulata, F. americana y A. plurijuga disminuyen con relación a la densidad de la madera (Figura 3a), además, se observa una tendencia al agrupamiento en las magnitudes de las retenciones a medida que la densidad aumenta, como es el caso para $A$. plurijuga. Al respecto, es importante mencionar que las retenciones correspondientes a un valor $\mathrm{R}=0$ (Figura 5a) se refieren a las probetas sin tratamiento, es decir, una concentración de boro igual a cero $(\mathrm{C}=0 \%)$. Por el contrario, en las tres especies del estudio, las retenciones aumentan en función de la concentración utilizada y, cabe señalar, que lo hacen con fuertes coeficientes de determinación $\left(\mathrm{R}^{2}=\right.$ 0,99) (Figura 4a). Estos resultados corroboran los reportados por Toker et al. (2009) para maderas de Pinus brutia $\left(\rho_{\mathrm{CH}}=532 \mathrm{~kg} \mathrm{~m}^{-3}\right)$ y Fagus orientalis $\left(\rho_{\mathrm{CH}}=520 \mathrm{~kg} \mathrm{~m}^{-3}\right)$, sobre las cuales también se impregnaron concentraciones similares de sales de boro. Igualmente, las magnitudes de las retenciones, considerando las concentraciones utilizadas en esta investigación, son del mismo orden que las encontradas por Temiz et al. (2008) para madera de Alnus glutinosa $\left(\rho_{\mathrm{CH}}=503 \mathrm{~kg} \mathrm{~m}^{-3}\right)$ y Pinus sylvestris $\left(\rho_{\mathrm{CH}}=520 \mathrm{~kg} \mathrm{~m}^{-3}\right)$.

\section{Figura 3}

Dispersiones de a), la retención (R); b), del tiempo de ignición (tign); y c), de la pérdida de masa ( $\Delta m$ ), en función de la densidad aparente $\left(\rho_{\mathrm{CH}}\right)$ para las concentraciones de $1 \%, 2 \%$ y $3 \%$ de sales de boro
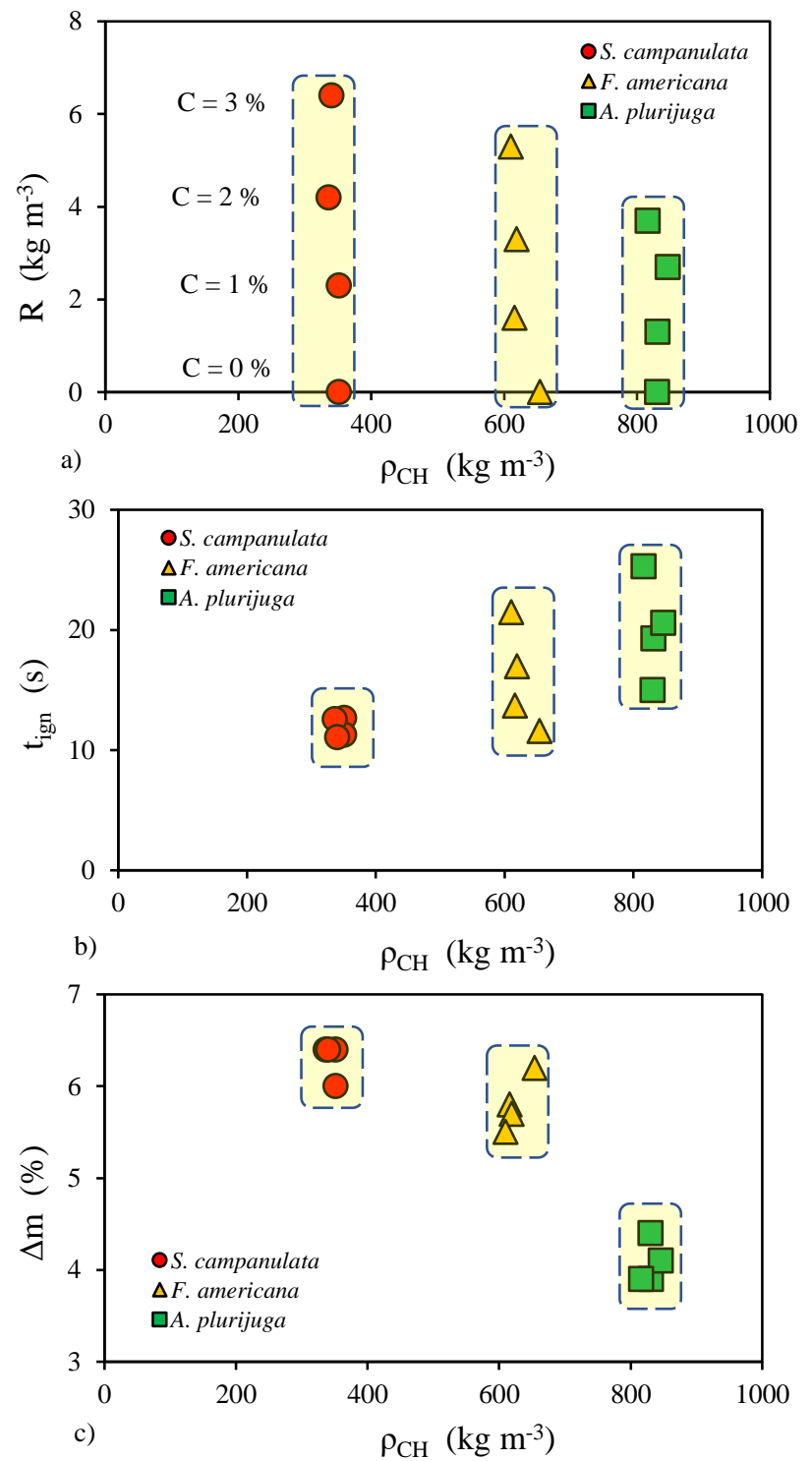

Fuente: Elaboración propia 
Figura 4

Correlaciones y coeficientes de determinación $\left(R^{2}\right)$ : de a) la retención $\left.(R) ; b\right)$ del tiempo de ignición (tign); y c) de la pérdida de masa ( $\Delta \mathrm{m})$, en función de las concentraciones de 1\%, $2 \%$ y $3 \%$ de sales de boro
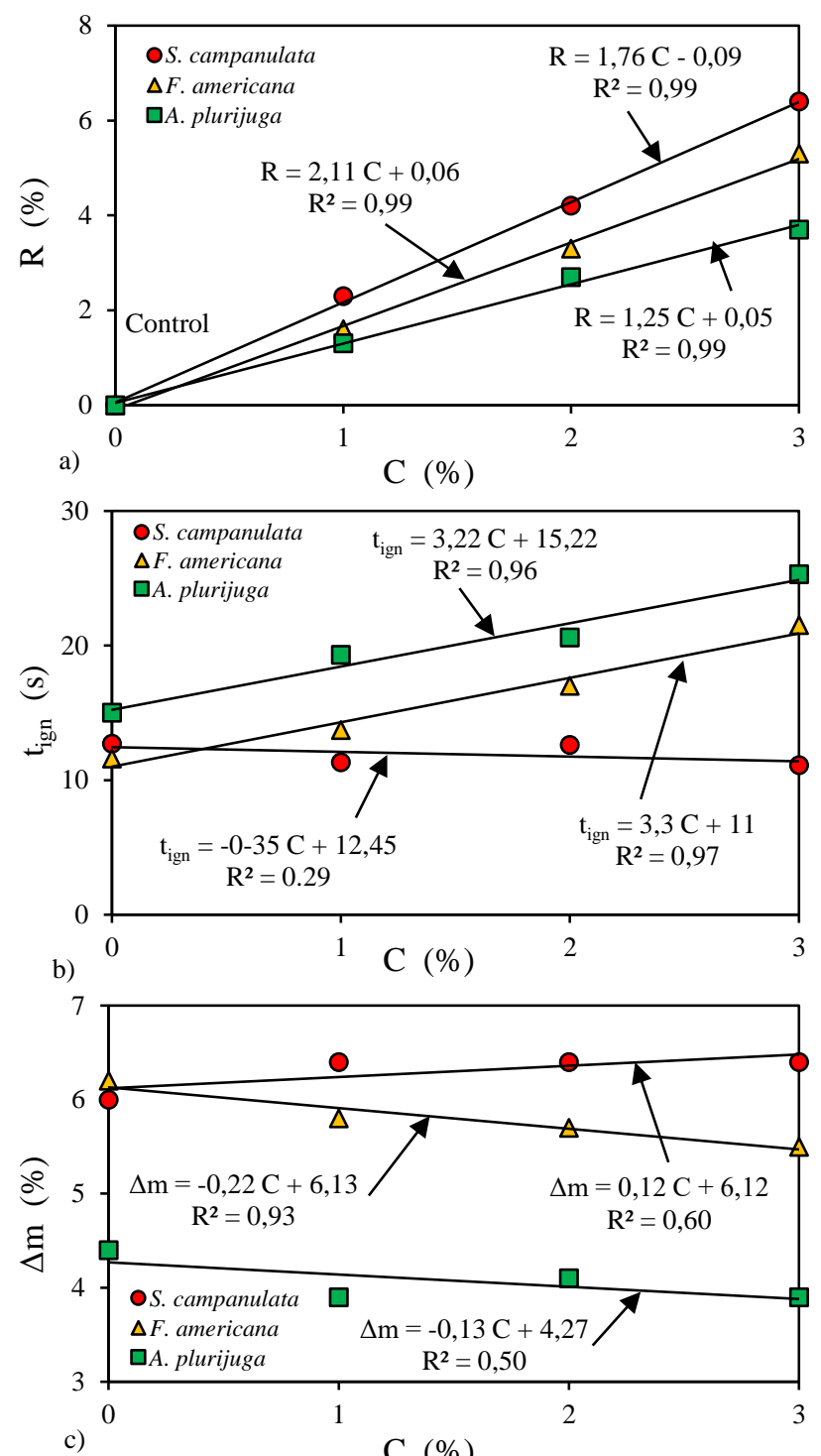

Fuente: Elaboración propia

Figura 5

Dispersiones de los tiempos de ignición ( $\left.t_{\text {ign }}\right)$ en función de las densidades aparentes $\left(\rho_{C H}\right)$ y sus correlaciones, detalladas en la Tabla 4

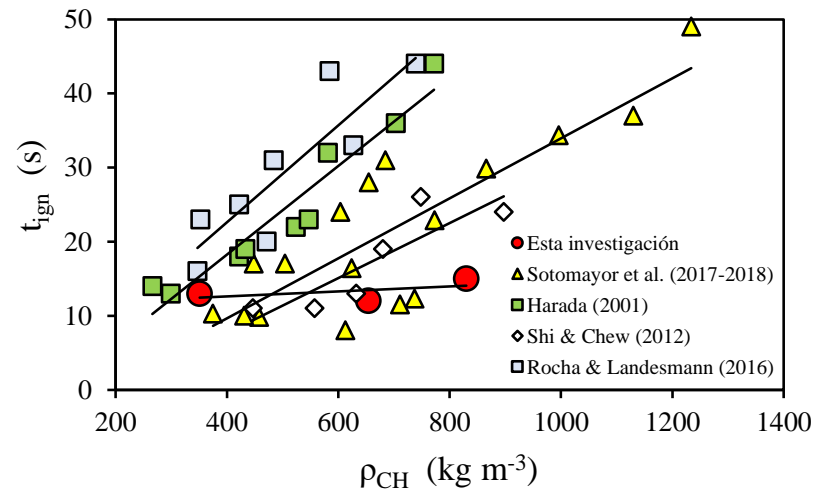

Fuente: Elaboración propia 


\section{Tiempo de ignición}

Los tiempos promedio de ignición medidos, para S. campanulata, F. americana y A. plurijuga (Tabla 1), son similares a los reportados en la bibliografía (Tabla 5) para maderas mexicanas cuyos protocolos experimentales fueron similares a los utilizados en esta investigación (Sotomayor \& Carrillo, 2017; Sotomayor et al., 2018; Sotomayor \& Gallegos, 2018; Sotomayor \& Giraldo, 2017; Sotomayor \& Osvaldová, 2017). Sin embargo, a diferencia de lo reportado en la bibliografía, en la madera no impregnada, no se observa una tendencia al aumento del tiempo de ignición a medida que la densidad de la madera aumenta (Harada, 2001; Shi \& Chew, 2019; Rocha \& Landesmann, 2016). Estas tendencias se muestran en la Figura 3 b con modelos lineales empíricos (Tabla 4), calculados a partir de los resultados obtenidos en esta investigación y de la bibliografía (ambos enlistados en la Tabla 5). El coeficiente de determinación de la correlación $\mathrm{t}_{\text {ign }}=\mathrm{f}\left(\rho_{\mathrm{CH}}\right)$ se calculó con los resultados de esta investigación (Tabla 4); su resultado estableció que este es 64 \% menor, en promedio, en comparación con los coeficientes $\mathrm{R}^{2}$ de la bibliografía.

Tabla 4

Correlaciones de las Figuras 5 y 6

\begin{tabular}{|l|c|c|}
\hline \multicolumn{1}{|c|}{ Autor } & Correlación $\mathbf{t}_{\text {ign }}=\mathbf{f}\left(\boldsymbol{\rho}_{\mathbf{C H}}\right)$ & $\mathbf{R}^{\mathbf{2}}$ \\
\hline Esta investigación (2019) & $\mathrm{t}_{\text {ign }}=0,0034 \rho_{\mathrm{CH}}+11,28$ & 0,28 \\
\hline Sotomayor et al. (2017-2018) & $\mathrm{t}_{\mathrm{ign}}=0,041 \rho_{\mathrm{CH}}-6,54$ & 0,71 \\
\hline Shi y Chew (2012) & $\mathrm{t}_{\mathrm{ign}}=0,037 \rho_{\mathrm{CH}}-7,18$ & 0,75 \\
\hline Rocha y Landesmann (2016) & $\mathrm{t}_{\mathrm{ign}}=0,065 \rho_{\mathrm{CH}}-3,53$ & 0,76 \\
\hline Harada (2001) Autor & $\mathrm{t}_{\mathrm{ign}}=0,059 \rho_{\mathrm{CH}}-5,66$ & 0,92 \\
\hline \multicolumn{1}{|c|}{ Correlación $\Delta \mathrm{m}=\mathrm{f}\left(\rho_{\mathrm{CH}}\right)$} & $\mathrm{R}^{2}$ \\
\hline Esta investigación (2019) & $\Delta \mathrm{m}=-0,0029 \rho_{\mathrm{CH}}+7,31$ & 0,51 \\
\hline Sotomayor et al. (2017-2018) & $\Delta \mathrm{m}=-0,019 \rho_{\mathrm{CH}}+27,12$ & 0,89 \\
\hline Sotomayor y Carrillo (2017) & $\Delta \mathrm{m}=-0,0085 \rho_{\mathrm{CH}}+10,15$ & 0,94 \\
\hline Sotomayor y Gallegos (2018) & $\Delta \mathrm{m}=-0,0244 \rho_{\mathrm{CH}}+322,46$ & 0,85 \\
\hline tign $=$ Tiempo de ignición; $\Delta \mathrm{m}=$ Pérdida de masa; $\rho_{\mathrm{CH}}=$ Densidad; $\mathrm{R}^{2}=$ Coeficiente de determinación. \\
\hline
\end{tabular}

Fuente: Elaboración propia

\section{Tabla 5}

Densidades aparentes, tiempos de ignición y pérdidas de masa para maderas no impregnadas en la bibliografía

\begin{tabular}{|c|c|c|c|c|c|c|}
\hline Especies mexicanas & $\rho_{\mathrm{CH}}$ & $t_{\text {ign }}$ & $\Delta \mathbf{m}$ & Especies internacionales & $\rho_{\mathrm{CH}}$ & $\mathbf{t}_{\text {ign }}$ \\
\hline & $\left(\mathrm{kg} \mathrm{m}^{-3}\right)$ & (s) & $(\%)$ & & $\left(\mathrm{kg} \mathrm{m}^{-3}\right)$ & (s) \\
\hline \multicolumn{4}{|c|}{ Sotomayor y Carrillo (2017) } & \multicolumn{3}{|c|}{ Harada (2001) } \\
\hline Tabebuia rosea & 613 & 8 & 5,3 & Cryptomeria japonica & 299 & 13 \\
\hline Andira inermis & 737 & 12 & 3,6 & Thujopsis dolabrata & 422 & 18 \\
\hline Juglans pyriformis & 773 & 23 & 3,5 & Pinus densiflora & 433 & 19 \\
\hline Quercus spp, & 866 & 30 & 2,6 & Larix leptolepsis & 524 & 22 \\
\hline Cordia elaeagnoides & 996 & 34 & 2,0 & Paulownia tomentosa & 266 & 14 \\
\hline \multicolumn{4}{|c|}{ Sotomayor et al, (2018) } & Juglans soeboldiana & 547 & 23 \\
\hline Tabebuia donnell-smithii & 448 & 17 & 20,8 & Fagus crenata & 581 & 32 \\
\hline Enterolobium cyclocarpum & 504 & 17 & 18,7 & Zelkova serrata & 703 & 36 \\
\hline Tabebuia rosea & 604 & 24 & 15,9 & Quercus mongolica & 772 & 44 \\
\hline Swietenia humilis & 655 & 28 & 14,9 & \multicolumn{3}{|c|}{ Shi y Chew (2012) } \\
\hline Lysiloma acapulcensis & 685 & 31 & 10,4 & Pinus contorta & 447 & 11 \\
\hline Cordia elaeagnoides & 1130 & 37 & 7,8 & Fagus grandifolia & 632 & 13 \\
\hline Tabebuia chrysantha & 1234 & 49 & 4,2 & Prunus serotina & 558 & 11 \\
\hline \multicolumn{4}{|c|}{ Sotomayor y Gallegos (2018) } & Quercus rubra & 897 & 24 \\
\hline Enterolobium cyclocarpum & 458 & 10 & 11,8 & Acer nigrum & 748 & 26 \\
\hline Tabebuia rosea & 624 & 16 & 5,7 & Fraxinus, americana & 680 & 19 \\
\hline Juniperus pyriformis & 711 & 12 & 6,1 & \multicolumn{3}{|c|}{ Rocha y Landesmann (2016) } \\
\hline \multicolumn{4}{|c|}{ Sotomayor y Osvaldová (2017) } & Joannesia princeps & 352 & 23 \\
\hline Abies religiosa & 431 & 10 & 5,4 & Cedrela, lilloi & 422 & 25 \\
\hline \multicolumn{4}{|c|}{ Sotomayor y Giraldo (2017) } & Schizolobium parahyba & 347 & 16 \\
\hline Gyrocaupus americanus & 375 & 10 & 9,0 & Ocotea porosa & 584 & 43 \\
\hline- & - & - & - & Capsicodendron dinisii & 471 & 20 \\
\hline- & - & - & - & Peltophorum dubium & 627 & 33 \\
\hline- & - & - & - & Zanthoxylum chiloperone & 739 & 44 \\
\hline- & - & - & - & Pinus caribaea & 484 & 31 \\
\hline
\end{tabular}

Fuente: Elaboración propia 
Los tiempos de ignición, para S. campanulata, F. americana y A. plurijuga, aumentan a medida que la densidad de la madera se incrementa, tanto para el grupo de referencia $(\mathrm{C}=0 \%)$ como para las tres concentraciones de sales de boro (Figura $3 b$ ). Se observa igualmente una tendencia a la dispersión de los valores del tiempo de ignición a medida que la concentración aumenta. Además, los tiempos de ignición, para $F$. americana y A. plurijuga, aumentan conforme la concentración de sales de boro se incrementa, con fuertes coeficientes de determinación (Figura 4b). Estos resultados coinciden con los reportados por Toker et al. (2009) para maderas de Pinus brutia $\left(\rho_{\mathrm{CH}}=532 \mathrm{~kg} \mathrm{~m}^{-3}\right.$ ) y Fagus orientalis $\left(\rho_{\mathrm{CH}}=520 \mathrm{~kg} \mathrm{~m}^{-3}\right)$, las cuales se impregnaron con concentraciones de sales de boro similares a las que se utilizaron en esta investigación. S. campanulata es un caso diferente, ya que muestra una ligera tendencia a disminuir del tiempo de ignición y un coeficiente de determinación muy débil.

\section{Pérdida de masa}

Las magnitudes de la pérdida de masa observadas en $S$. campanulata, $F$. americana y A. plurijuga (Tabla 1) son similares a las que se reportan en la bibliografía para maderas mexicanas (Tabla 5). Las pruebas de normalidad en las distribuciones de las muestras de las tres especies, así como de las tres concentraciones de sales de boro y del grupo de control $(\mathrm{C}=0 \%)$ fueron normales. Igualmente, para cada especie, en las pruebas de verificación y análisis de varianza hay diferencias estadísticamente significativas entre el grupo de control y las tres concentraciones estudiadas (Tabla 3). Sin embargo, con excepción de $S$. campanulata, tanto las densidades como los tiempos de ignición y la pérdida de masa resultaron no homogéneos entre las cuatro concentraciones.

La Figura 3c evidencia que la pérdida de masa disminuye a medida que la densidad de la madera aumenta (Figura 3c). Debido a que el protocolo experimental utilizado en esta investigación es diferente al reportado en la bibliografía, su contrastación es compleja. Sin embargo, esta tendencia coincide con las que se muestran en la Figura 6. En la Tabla 4 se detallan las correlaciones y coeficientes de determinación, así como las correlaciones lineales construidas con datos recolectados en la literatura (Tabla 5).

Estos corolarios coinciden con los de Toker et al. (2009) para maderas de Pinus brutia $\left(\rho_{\mathrm{CH}}=\right.$ $532 \mathrm{~kg} \mathrm{~m}^{-3}$ ) y Fagus orientalis $\left(\rho_{\mathrm{CH}}=520 \mathrm{~kg} \mathrm{~m}^{-3}\right.$ ), impregnadas con concentraciones de sales de boro similares a las que se utilizaron en esta investigación. A partir de esta coincidencia se deduce que la pérdida de masa disminuye cuando la densidad de la madera aumenta. El coeficiente de determinación correspondiente a la correlación calculada, tomando como base los resultados de esta investigación (Tabla 4), es en promedio $57 \%$ menor que los coeficientes reportados por los autores mencionados en esta tabla. Respecto a la pérdida de masa en función de la concentración de sales de boro, para $F$. americana y A. plurijuga, se observan ligeras tendencias a la disminución. Un caso diferente muestra S. campanulata, cuya regresión es positiva (Figura 4c).

Figura 6

Dispersiones de la pérdida de masa $(\Delta m)$ en función de las densidades aparentes $\left(\rho_{C H}\right)$ y sus correlaciones, detalladas en la Tabla 4

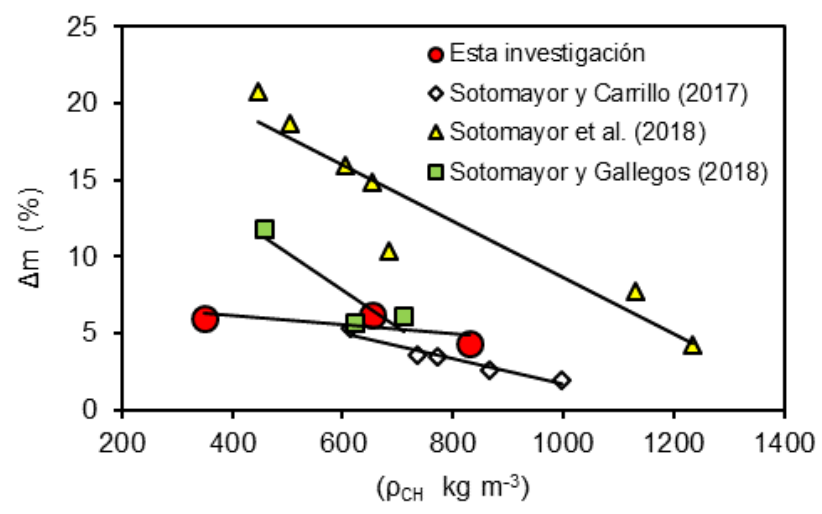

Fuente: Elaboración propia 


\section{Conclusiones}

El método baño caliente-frío permite impregnar con sales de boro las maderas de S. campanulata, F. americana y A. plurijuga. El tratamiento con concentraciones de $1 \%, 2 \%$ y $3 \%$ no altera de manera significativa las densidades de estas tres especies. El impregnado aumenta la resistencia aparente de la madera a la exposición al fuego. Además, retarda los tiempos de ignición y disminuye la pérdida de masa cuando la madera es expuesta al fuego en condiciones de laboratorio.

La madera de S. campanulata impregnada con las sales de boro al $3 \%$ presenta las retenciones $(6,4 \mathrm{~kg} \mathrm{~m}-3)$ y la pérdida de masa $(6,4 \%)$ más altas y el tiempo de ignición menor $(11,1 \mathrm{~s})$.

Con todo, las magnitudes de las retenciones, los tiempos de ignición y la pérdida de masa son diferentes para cada especie y concentración. Las retenciones fueron proporcionales a la concentración e inversamente proporcional a la densidad. Los tiempos de ignición se incrementan a medida que la densidad de la madera aumenta y la concentración de sales de boro aumenta. La pérdida de masa disminuye en función de la densidad aparente de la madera. No se observa tendencias claras respecto a la pérdida de masa en función de la concentración de sales de boro. Estos resultados encuentren aplicación práctica en la selección e impregnado de la madera para su uso en la industria de la construcción.

\section{Referencias}

Ali, S., Hussain, S. A., and Tohir, M. Z. M. (2019). Fire test and effects of fire retardant on the natural ability of timber: a review. Pertanika Journal of Science \& Technology, (27), 867-895. http://psasir.upm.edu.my/id/eprint/68698/

Atar, M., Keskin, H., Korkut, S., and Korkut, D. S. (2011). Impact of impregnation with boron compounds on combustion properties of oriental beech (Fagus orientalis Lipsky) and varnishes. African Journal of Biotechnology, (10), 2867-2874. https://doi.org/10.5897/AJB10.1567

Ávila, L. E. A., Herrera, M. A., y Raya, D. (2012). Preservación de la madera en México. México: Universidad Michoacana de San Nicolás de Hidalgo.

Babrauskas, V. (2002). Ignition of wood: A review of the state of the art. Journal of Fire Protection Engineering, (12), 163-189. DOI: 10.1177/10423910260620482

Bartlett, A. I., Hadden, R. M., \& Bisby, L. A. (2019). A review of factors affecting the burning behaviour of wood for application to tall timber construction. Fire Technology, (55), 1-59. DOI: /10.1007/s10694018-0787-y

Boonmee, N., \& Quintiere, J. G. (2002). Glowing and flaming autoignition of wood. Proceedings of the Combustion Institute, (29), 289-296. DOI: 10.1016/S1540-7489(02)80039-6

Friquin, K. (2011). Material properties and external factors influencing the charring rate of solid wood and gluelaminated timber. Fire and Materials, (35), 303-327. DOI: 10.1002/fam.1055

Gaff, M., Kačík, F., Gašparík, M., Todaro, L., Jones, D., Corleto, R., Osvaldová, L. M., \& Čekovská, H. (2019). The effect of synthetic and natural fire-retardants on burning and chemical characteristics of thermally modified teak (Tectona grandis L. f.) wood. Construction and Building Materials. (200), 551-558. DOI: 10.1016/j.conbuildmat.2018.12.106

Harada, T. (2001). Time to ignition, heat release rate and fire endurance time of wood in cone calorimeter test. Fire and Materials, (25), 161-167. DOI: 10.1002/fam.766

International Organization for Standardization, Geneva (2012). ISO 3129:2012. Wood. Sampling methods and general requirements for physical and mechanical testing of small clear wood specimens. https://www.iso.org/standard/52489.html 
International Organization for Standardization, Geneva (2014a). ISO 13061-1:2014. Physical and mechanical properties of wood. Test methods for small clear wood specimens. Part 1: Wood. Determination of moisture content for physical and mechanical tests. https://www.iso.org/standard/60063.html

International Organization for Standardization, Geneva (2014b). ISO 13061-2:2014. Wood. Physical and mechanical properties of wood. Test methods for small clear wood specimens. Part 2: Determination of density for physical and mechanical tests. https://www.iso.org/standard/60064.html

Jin, E., \& Chung, Y.J. (2018). Fire risk of wood treated with boron compounds by combustion test. Fire Science and Engineering, 32(3), 19-26. DOI: 10.7731/KIFSE.2018.32.3.019

Koumbi-Mounanga, T., Morris, P. I., Lee, M. J., Saadat, N. M., Leblon, B. \& Cooper, P. A. (2015). Prediction and evaluation of borate distribution in Eastern black spruce (Picea mariana var. mariana) wood products. Wood Science and Technology, (49), 457-473. DOI: 10.1007/s00226-015-0714-z

Organismo Nacional de Normalización y Certificación de la Construcción y Edificación. (2014). Norma Mexicana NMX-C-178- ONNCCE-2014 Industria de la Construcción. Preservadores para MaderaClasificación y Requisitos.

https://www.onncce.org.mx/es/?option=com_merchant\&view=category\&cid=24

Osvaldová, L. M., Gaspercova, S., Mitrenga, P., \& Osvald, A. (2016). The influence of density of test specimens on the quality assessment of retarding effects of fire retardants. Wood Research, (61), 35-42. http://www.centrumdp.sk/wr/201601/04.pdf

Osvaldová, L. M. (2018). Density of test bodies and its effect on burning rate of fire-retardant treated samples. MATEC Web of Conferences, (213), 1-4. DOI: 10.1051/matecconf/201821303002

Östman, B. A. L. (2017). Fire performance of wood products and timber structures. International Wood Products Journal, (8), 74-79. DOI: 10.1080/20426445.2017.1320851

Pereira, E. E., Mota, A., Cabral, J., Campos, T., Chaves, M. D., \& Benigno, J. (2017). Influence of boron compounds on the physical properties of Eucalyptus sp. wood. Scientia Forestalis, (45), 197-204. https://www.ipef.br/publicacoes/scientia/nr113/cap20.pdf

Ragan, B., Kačíková, D., \& Pauld’uro, M. (2016). Influence of physical and chemical characteristics of selected tree species on mass loss and rate of burning after exposure to radiant heating. Acta Facultatis Xylologiae Zvolen, (58), 121-131. DOI: 10.17423/afx.2016.58.2.13

Rocha, M. A., \& Landesmann, A. (2016). Combustion properties of Brazilian natural wood species. Fire and Materials, (40), 219-228. DOI: 10.1002/fam.2281

Shi, L., \& Chew, M. Y. L. (2019). Experimental study of woods under external heat flux by autoignition: Ignition time and mass loss rate. Journal of Thermal Analysis and Calorimetry, (111), 1399-1407. DOI: $10.1007 / \mathrm{s} 10973-012-2489-\mathrm{x}$

Silva, J. A., Fuentes, F. J., Rodríguez, R., Torre,s P. A., Lomelí, M. A., Ramos, J., Waitkus, C., y Richter, H. G. (2010). Fichas de propiedades tecnológicas y usos de maderas nativas de México e importadas. México: Comisión Nacional Forestal.

Simsek, H., Baysal, E., Yilmaz, M., \& Culha, F. (2013). Some mechanical properties of wood impregnated with environmentally-friendly boron and copper based chemicals. Wood Research. (58), 495-504. DOI: 10.1016/j.conbuildmat.2010.04.028

Sogutlu, C., Demirci, Z., Dongel, N., Ozgur Imirzi, H., Doruk, S., \& Yalınkılıc, A. C. (2011). The determination of the resistance to burning of some wood types impregnated with sodium borate solution. Wood Research, (56), 233-244. Disponible en: http://www.woodresearch.sk/wr/201102/09.pdf

Sotomayor, J. R. (2015). Banco FITECMA de características físico-mecánicas de maderas mexicanas. México: Universidad Michoacana de San Nicolás de Hidalgo. https://www.researchgate.net/ 
Sotomayor, J. R., y Ávila, L. E. A. (2019). Retención y penetración de sales de boro en tres maderas angiospermas: Spathodea campanulata, Fraxinus americana y Albizia plurijuga. Revista Ciencia y Tecnología UTEQ, 12(2), 23-31. DOI: 10.18779/cyt.v12i2.323.

Sotomayor, J. R., y Carrillo, M. I. (2017). Comportamiento al fuego de cinco especies mexicanas. Estudio comparativo. Investigación e Ingeniería de la Madera, (13), 4-38. https://www.researchgate.net/

Sotomayor, J. R., y Correa, S. (2016). Retención de sales de boro en la madera y su efecto en el módulo de elasticidad dinámico. Revista Científica, (24), 1-19. DOI:

10.14483/10.14483/udistrital.jour.RC.2016.24.a9

Sotomayor, J. R., y Gallegos, G. (2018). Reacción al fuego de madera sólida y laminada de Enterolobium cyclocarpum, Tabebuia rosea y Juniperus pyriformis. Estudio comparativo. Investigación e Ingeniería de la Madera, (14), 39-78. https://www.researchgate.net/

Sotomayor, J. R., \& Giraldo, M. P. (2017). Resistencia al fuego de madera densificada. Investigación e Ingeniería de la Madera, (13), 45-62. https://www.researchgate.net/

Sotomayor, J. R., Giraldo, M. P, Haurie, L., Lacasta, A. M., Montón, J., Palumbo, M., \& Navarro, A. (2018). Characterization of the fire behaviour of tropical wood species for use in the construction industry. Investigación e Ingeniería de la Madera, (14), 4-24. https://www.researchgate.net/

Sotomayor, J. R., y Osvaldová, L. M. (2017). Resistencia al fuego de madera laminada. Investigación e Ingeniería de la Madera, (13), 4-21. https://www.researchgate.net/

Sotomayor, J. R., \& Villaseñor, J. M. (2016). Retención y absorción de solución de sales de boro de diez maderas mexicanas. Acta Universitaria, (26), 12-19. DOI: 10.15174/au.2016.835

Tamarit, J. C., y López, J. L. (2007). Xilotecnología de los principales árboles tropicales de México. México: Instituto Nacional de Investigaciones Forestales, Agrícolas y Pecuarias.

Temiz, A., Geze,r E. G., Yildiz, U. C., \& Yildiz, S. (2008). Combustion properties of alder (Alnus glutinosa L.) Gaertn. subsp. barbata (C.A. Mey) Yalt.) and southern pine (Pinus sylvestris L.) wood treated with boron compounds. Construction and Building Materials, (22), 2165-2169. DOI: 10.1016/j.conbuildmat.2007.08.011

Toker, H., Baysal, E., Ozcifci, A., Simsek, H., Altun, S., Yapici, F., \& Goktas, O. (2009). Some combustion parameters of wood impregnated with borates. Forest Products Journal, 59(6), 85-89. https://search.proquest.com/openview/e54ab24b7db695f5c6da7a7dff261562/1?pqorigsite $=$ gscholar $\&$ cbl $=25222$

Tondi, G., Wieland, S., Wimmer. T., Thevenon, M. F., Pizzi, A., \& Petutschnigg, A. (2012). Tannin-boron preservatives for wood buildings: mechanical and fire properties. European Journal of Wood Products, (70), 689-696. DOI 10.1007/s00107-012-0603-1

Uner, I. H., Deveci, I., Baysal, E., Turkoglu, T., Toker, H., \& Peker, H. (2016). Thermal analysis of oriental beech wood treated with some borates as fire retardants. Maderas. Ciencia y tecnología, (18), 293-304. DOI: $10.4067 /$ S0718-221X2016005000027

Wang, F., Liu, J., \& Lv, W. (2017). Thermal degradation and fire performance of wood treated with PMUF resin and boron compounds. Fire and Materials, (41), 1051-1057. DOI: 10.1002/fam.2445

Xu, Q. F., Chen, L. Z., Harries, K. A., Zhang, F. W., Liu, Q., \& Feng, J. H. (2015). Combustion and charring properties of five common constructional wood species from cone calorimeter tests. Construction and Building Materials, (96), 416-427. DOI: 10.1016/j.conbuildmat.2015.08.062 